\title{
Experimental investigation of the influence of internal frames on the vibroacoustic behavior of a stiffened cylindrical shell using wavenumber analysis
}

\author{
V. Meyer ${ }^{\mathrm{a}, \mathrm{b}, *}$, L. Maxit $^{\mathrm{a}}$, Y. Renou ${ }^{\mathrm{b}}$, C.Audoly $^{\mathrm{b}}$ \\ ${ }^{a}$ Univ Lyon, INSA-Lyon, Laboratoire Vibrations Acoustique, F-69621 Villeurbanne, France \\ ${ }^{b}$ DCNS Research, 199 avenue Pierre-Gilles de Gennes, 83190 Ollioules, France
}

\begin{abstract}
The understanding of the influence of non-axisymmetric internal frames on the vibroacoustic behavior of a stiffened cylindrical shell is of high interest for the naval or aeronautic industries. Several numerical studies have shown that the non-axisymmetric internal frame can increase the radiation efficiency significantly in the case of a mechanical point force. However, less attention has been paid to the experimental verification of this statement. That is why this paper proposes to compare the radiation efficiency estimated experimentally for a stiffened cylindrical shell with and without internal frames. The experimental process is based on scanning laser vibrometer measurements of the vibrations on the surface of the shell. A transform of the vibratory field in the wavenumber domain is then performed. It allows estimating the far-field radiated pressure with the stationary phase theorem. An increase of the radiation efficiency is observed in the low frequencies. Analysis of the velocity field in the physical and wavenumber spaces allows highlighting the coupling of the circumferential orders at the origin of the increase in the radiation efficiency.
\end{abstract}

Keywords: Stiffened shell, Vibroacoustics, Non-axisymmetric, Laser vibrometer, wavenumber analysis.

\section{Introduction}

Many works can be found in the literature dealing with the vibroacoustic behavior of cylindrical shells reinforced by circumferential stiffeners [1, 2, 3, As a matter of fact, many structures in the aeronautical or naval industry can be 5 modeled by stiffened cylindrical shells. Williams et al. 4] measured the nearfield pressure on a submerged cylinder excited by a point force. They deduced the vibration and radiation from the cylinder thanks to a technique called the generalized nearfield acoustical holography. In a subsequent study, the authors

\footnotetext{
* Corresponding author

Email address: valentin.meyer@dcnsgroup.com (V. Meyer)
}

Preprint submitted to Mechanical Systems and Signal Processing 
analyzed the results in the wavenumber domain [5]. This analysis shows a great interest to give a physical insight of the vibroacoustic behavior of the cylindrical shell. More particularly, wavenumber diagrams can be plotted and the waves contributing to the far-field radiation can be identified. The technique is later used by Photiadis et al. to investigate the response of a stiffened cylinder in the wavenumber domain [6]. The wavenumber diagrams show more complex 15 patterns, due to the stiffeners periodicity and the propagation of Bloch-Floquet waves. Slowness surfaces, which are given by the normal to the curves of large amplitude and large radius of curvature on the wavenumber diagrams, are used to predict the dominant directions of wave propagation.

The studies mentioned previously are limited to axisymmetric systems. How20 ever, in industrial applications, internal structures such as floor partitions or engine foundations can have an influence on the vibroacoustic behavior of the stiffened cylindrical shell. Experimental work has been carried out for a stiffened cylindrical shell coupled to numerous oscillators, and the influence on the scattered or radiated pressure has been highlighted [7, 8, The results show

25 that adding internal degrees of freedom reduces scattering from flexural BlochFloquet waves and gives rise to a noisy speckle pattern. Nevertheless, in the case of a point mechanical excitation, only a few studies based on numerical simulation have investigated the effect of non-axisymmetric internal frames. Some of them use discretization methods such as Finite Element Method and Boundary

30 Element Method to predict the radiated pressure by an immersed shell with and without internal masses or structures and show the importance of flexible mounts 9, 10. Other studies use substructuring approaches based on the admittance method to include internal structures in an axisymmetric cylindrical shell [11, 12. The main results are that more circumferential orders tend to 35 play a role in the response of the cylindrical shell when it is coupled to a nonaxisymmetric internal frame. If the problem is decomposed on a modal basis, it is equivalent to say that the non-axisymmetry tends to couple the circumferential orders [13. As the structural waves having low wavenumbers are the main contributors to the far-field radiated pressure, the coupling with low circumfer-

40 ential numbers tends to increase the radiation efficiency. Another effect of the internal frame is given by the fuzzy internal theory [14, 15]. This theory states that if the internal structure has a high modal density and if the impedance at the junction with the cylindrical shell is adapted, the addition of internal degrees of freedom can give rise to an apparent damping effect.

45 The aim of the present work is to discuss experimental results of a pointdriven stiffened cylindrical shell with and without a non-axisymmetric internal frame. The effect of the internal structure on the vibroacoustic behavior of the cylindrical shell, and more especially on the radiation efficiency is investigated. The main obstacle for estimating accurately the radiation efficiency

50 lies in the estimation of the radiated power. Precision methods using sound pressure require a large number of microphones and are difficult to set up [16]. Measurement by scanning using sound intensity [17] is widely used to measure the radiated power from machines, but it can be difficult to define an enclosing surface around the system. To tackle these issues, the methodology of the 
present work is based on the use of the stationary phase theorem. It relates the far-field radiated pressure to the spectral velocity field of the shell (i.e. the velocity field in the wavenumber domain). An advantage of this approach over the measurement with microphones or intensity probes is that the directivity can also be estimated. The spectral velocity field can be obtained with a scanning 60 laser vibrometer and a 2D Fourier transform. Attention is paid to the definition of the scanning grid in order to avoid aliasing effect.

The paper is organized as follows:

- Background on the estimation of the far-field radiated pressure from the spectral displacements of the shell is given in section 2 .

- The system and the experimental setup are presented in section 3 .

- Operational dispersion curves plotted from the spectral velocity field are discussed in section 4

- In section 5 the stationary phase theorem is used to determine the far-field radiated power and to investigate the influence of the non-axisymmetric frame on the radiation efficiency.

- Velocity fields are compared for the axisymmetric and non-axisymmetric cases in the real space and in the wavenumber domain in section 6 .

- Finally, conclusions are drawn in section 7 .

\section{Spectral displacements and far-field pressure}

75

Let us consider a cylindrical shell of radius $R_{0}$ immersed in an acoustic domain. The shell can either be of infinite length or of finite length extended by a cylindrical baffle. Considering the cylindrical coordinates $(x, r, \theta), x$ being the axis of the shell, $W(x, \theta)$ is the radial shell displacements at a given angular frequency $\omega$.

80 A two dimensional Fourier transform can be used to transform the spatial variations of a vibratory field into the wavenumber domain. The $2 \mathrm{D}$ Fourier transform of $W(x, \theta)$ is given by

$$
W(x, \theta) \mapsto \tilde{\tilde{W}}\left(k_{x}, n\right)=\int_{\mathbb{R}} \int_{\theta=0}^{2 \pi} W(x, \theta) e^{-\mathrm{j}\left(k_{x} x+n \theta\right)} \mathrm{d} x \mathrm{~d} \theta
$$

where $k_{x}$ is the axial wavenumber and $n$ the circumferential order. As the cylindrical shell is $2 \pi$-periodic along the circumferential coordinate $\theta$, then $n$ is 85 a relative integer.

The wavenumber analysis gives an opportunity to analyze the wave propagation on the structure and the role played by the contribution of each circumferential order. The wavenumber analysis also gives an insight on the spectral 
components contributing to the radiated pressure, through the radiation circle defined by the points of coordinates $\left(\bar{k}_{x}, \bar{n}\right)$ in the wavenumber domain

$$
\left(\frac{\bar{n}}{R_{0}}\right)^{2}+\bar{k}_{x}^{2}=k_{0}^{2}
$$

where $k_{0}=\frac{\omega}{c_{0}}$ is the acoustic wavenumber and $c_{0}=340 \mathrm{~m} . \mathrm{s}^{-1}$ is the sound velocity in air. For a plate, the radiation circle represents the supersonic acoustic domain, in which the components radiate to the far-field. It remains a good indicator for localizing the radiating waves on a cylindrical shell when the effect of the curvature is low [5].

The vibratory field in the wavenumber domain can be used to estimate the far-field radiated pressure. For $\mathcal{R}$ in the acoustic far-field, i.e. $\mathcal{R} \gg \frac{2 \pi}{k_{0}}$, the asymptotic expression for the Hankel function and the stationary phase theorem can be used [18. If $\xi$ is the angle from the point to the normal axis and $\theta$ is the circumferential angle, the far-field pressure $p$ can be written as:

$$
p(\mathcal{R}, \xi, \theta)=\sum_{n=-\infty}^{+\infty} \frac{\mathrm{j} \rho_{0} \omega^{2}}{2 \pi^{2} \mathcal{R} k_{0} \cos \xi} \frac{\tilde{W}\left(-k_{0} \sin \xi, n\right)}{H_{n}^{(2)^{\prime}}\left(R_{0} k_{0} \cos \xi\right)} e^{-\mathrm{j} \mathcal{R} k_{0}+\mathrm{j} n\left(\theta+\frac{\pi}{2}\right)}
$$

where $\rho_{0}=1.2 \mathrm{~kg} \cdot \mathrm{m}^{-3}$ is the density of air and $H_{n}^{(2)^{\prime}}$ is the derivative of the Hankel function of second kind and order $n$. For a cylinder of finite length, the stationary phase theorem is based on the assumption of a baffled cylinder. However, the influence of the baffle on the radiation due to radial vibrations is weak when the shell is long compared to the acoustic wavelength 19]. The stationary phase theorem may then be used to predict the radiated pressure from the cylindrical shell. One of the advantages of the wavenumber analysis is that it allows the calculation of the radiated pressure from vibrations measurements only.

110 The far-field radiated power $P_{a}$ is obtained by integrating the radiated pressure over the surface of the sphere of radius $\mathcal{R}$ :

$$
P_{a}=\frac{1}{\rho_{0} c_{0}} \int_{\xi=0}^{\pi} \int_{\theta=0}^{2 \pi} p^{2}(\mathcal{R}, \xi, \theta) \mathcal{R}^{2} \sin \theta \mathrm{d} \xi \mathrm{d} \theta
$$

As the acoustic domain is conservative, any value of $\mathcal{R}$ in the far-field can be chosen to yield $P_{a}$. The radiation efficiency $\sigma$ of a cylindrical shell is defined by the ratio between the actual far-field radiated power $P_{a}$ and the power that would be radiated by a cylindrical shell of the same radiating surface $S=$ $2 \pi R_{0} L$, having an uniform radial velocity equal to the mean quadratic velocity $<\dot{W}>$ :

$$
\sigma=\frac{P_{a}}{\rho_{0} c_{0} S<\dot{W}>^{2}}
$$

The mean quadratic velocity is defined as follows:

$$
<\dot{W}>^{2}=\frac{1}{S} \int_{x=0}^{L} \int_{\theta=0}^{2 \pi}|\dot{W}(x, \theta)|^{2} \mathrm{~d} x R_{0} \mathrm{~d} \theta
$$

with $\dot{W}(x, \theta)$ the point radial velocity at the point of coordinates $\left(x, R_{0}, \theta\right)$. 


\section{Presentation of the experimental setup}

\subsection{Axisymmetric stiffened cylindrical shell}

A stiffened cylindrical shell, which geometrical characteristics are given in table 1. is shown in Fig. 1. The cylindrical shell is made of standard nonalloy steel, for which common values may be considered: Young's modulus $E=$

$1252.1 .10^{11} \mathrm{~Pa}$, density $\rho_{s}=7800 \mathrm{~kg} . \mathrm{m}^{-3}$ and Poisson's coefficient $\nu=0.3$. It has 72 identical stiffeners of rectangular cross-section, which have been machined from a thicker cylinder. The cylindrical shell is divided in five sections with three different stiffeners spacings, as shown in the sketch in Fig. 11. $15 \mathrm{~mm}$ thick caps are screwed at the ends of the cylinder. This stiffened cylindrical shell is referred to as the axisymmetric case.

Table 1: Stiffened cylindrical shell dimensions ( $\mathrm{mm})$.

\begin{tabular}{ccc}
\hline Parameter & Notation & Value \\
\hline Radius & $R_{0}$ & 100 \\
Length & $L$ & 1500 \\
Shell thickness & $h$ & 1.5 \\
Stiffeners height & $h_{w}$ & 5 \\
Stiffeners thickness & $t_{w}$ & 1 \\
Stiffeners spacing 1 & $\alpha$ & 24 \\
Stiffeners spacing 2 & $\beta$ & 20 \\
Stiffeners spacing 3 & $\gamma$ & 17 \\
\hline
\end{tabular}

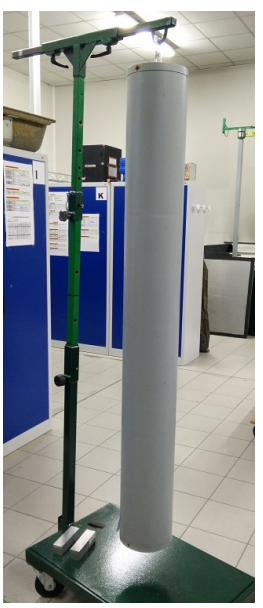

(a)

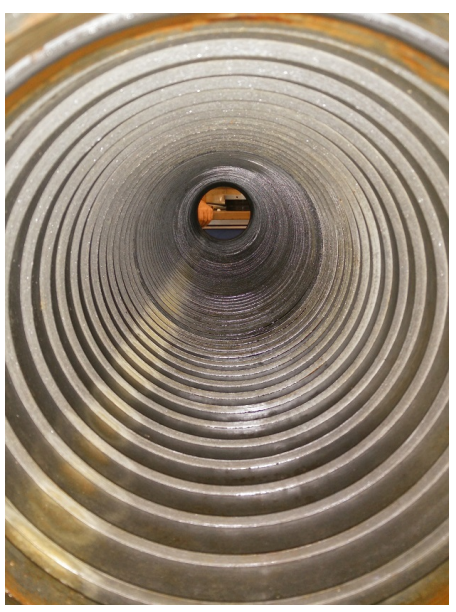

(b)

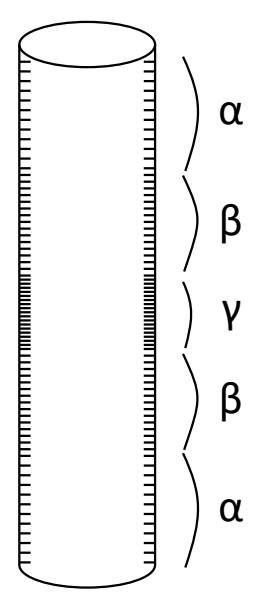

(c)

Figure 1: Pictures of the axisymmetric stiffened cylindrical shell: (a) hanged on an arm and (b) interior view. (c) Sketch of the cylindrical shell with the sections of different stiffeners spacings. 
The ring frequency $f_{r}$, for which the longitudinal wavelength is equal to the circumference, is defined by the following equation [18]:

$$
f_{r}=\frac{1}{2 \pi R_{0}} \sqrt{\frac{E}{\rho_{s}\left(1-\nu^{2}\right)}}=8657 \mathrm{~Hz} .
$$

The coincidence frequency, for which the acoustic wavenumber $k_{0}$ is equal to the flexural wavenumber in an equivalent plate of thickness $h$, can be defined as [18:

$$
f_{c}=\frac{c_{0}^{2}}{2 \pi h} \sqrt{\frac{12 \rho_{s}\left(1-\nu^{2}\right)}{E}}=7812 \mathrm{~Hz} .
$$

In general, the radiation efficiency defined in Eq. (5) presents peaks at the ring frequency and at the coincidence frequency 20. It can be noticed that for this model the ring frequency and the coincidence frequency have close values. The radiation efficiency is expected to be low below these frequencies and to tend to

\subsection{Non-axisymmetric internal frame}

In the second configuration, a non-axisymmetric internal frame is added inside the cylinder described in section 3.1. As shown in Fig. 2, it is a floor which is $869 \mathrm{~mm}$ long, $64.3 \mathrm{~mm}$ high and $170.6 \mathrm{~mm}$ wide. It consists in a $1.5 \mathrm{~mm}$ thick plate, for which the ends have been cut along a circular arc of radius $98.5 \mathrm{~mm}$, before being bended to form right angles. The contact between the cylindrical shell and the floor is only on these two circular arcs. A square tube of $20 \mathrm{~mm}$ wide and $2 \mathrm{~mm}$ thick in steel is glued under the horizontal part and reinforces the structure lengthwise.

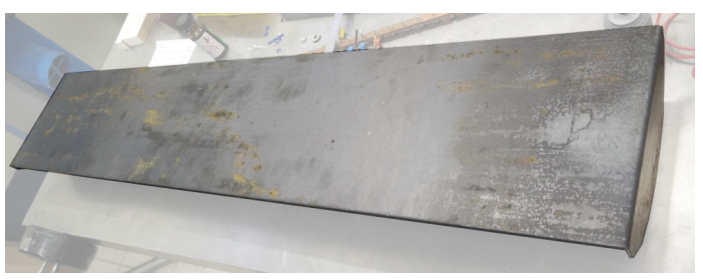

(a)

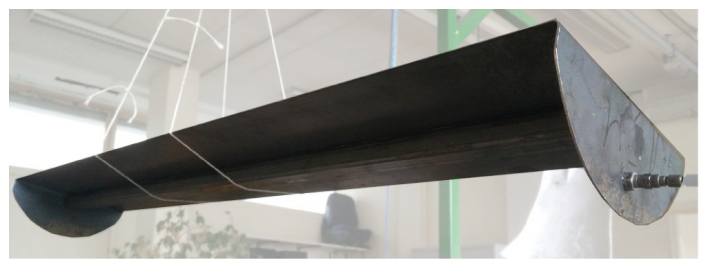

(b)

Figure 2: Pictures of the non-axisymmetric internal frame: (a) top view and (b) bottom view. 
The floor is placed inside the cylindrical shell, with line contacts along the circumference at $x=0.315 \mathrm{~m}$ and $x=1.185 \mathrm{~m}$. Several constraints need to be taken into account to assemble the two subsystems:

- the displacements continuity should be verified (i.e. rigid connection) and no damping (i.e. energy dissipation) should be introduced;

- the junctions should be strong enough to support the floor when the cylindrical shell is hanged;

- the access inside the cylindrical shell is difficult because of the small radius;

- the process should not damage the cylinder;

- the internal structure could be dismounted once the measurements done.

Tests are done on samples made of two steel plates perpendicularly assembled (not presented here for sake of brevity). Several types of glue, as well as brazing are compared. An acrylic glue with activator (Loctite AA 330) is chosen for this application. A picture of the assembled system is shown in Fig. 3.

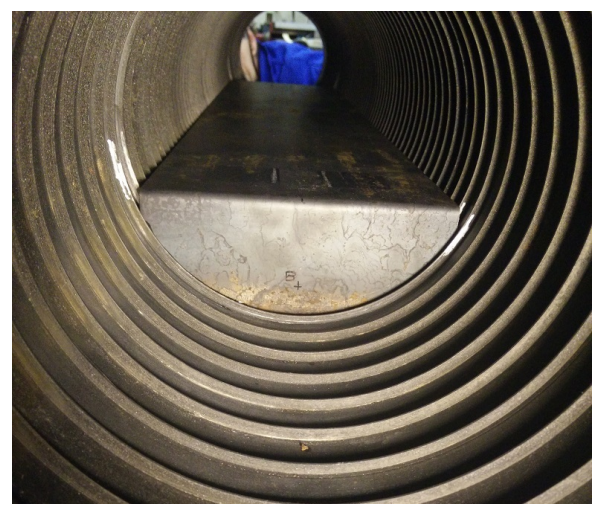

Figure 3: Interior view of the stiffened cylindrical shell with the non-axisymmetric internal frame.

\subsection{Experimental setup}

As shown in Fig. 4, the system is hanged vertically on an arm. A strap is used in order to limit the transfer of vibrations between the system and the arm. A rotating platform allows rotating the cylindrical shell around its axis and identifying precisely the angles. A $12.5 \mathrm{~mm}$ diameter patch is glued on the outer surface of the cylindrical shell, and screwed to a shaker. The shaker 170 is hanged on the rotating platform in order to excite always the same point of coordinate $(x, r, \theta)=(1.195,0.1,0)$ in the cylindrical system (with $x=0$ at the bottom of the cylindrical shell). This excitation point is located at the bottom of a stiffener, and lies at $10 \mathrm{~mm}$ from one of the floor junctions in 
the non-axisymmetric configuration. Two elements come in between the patch and the shaker: an impedance head that measures acceleration and force at the excitation point, and a threaded rod that limits the moments and allows assuming a radial excitation in the cylindrical system.

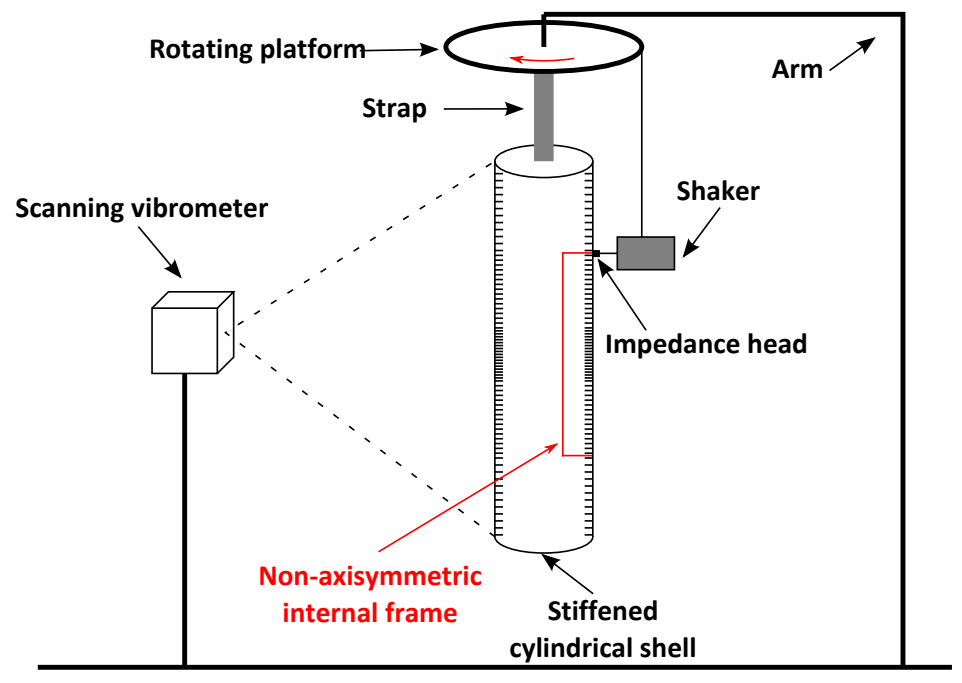

Figure 4: Sketch of the experimental setup.

The measurements are conducted in a semi-anechoic room $(8.6 \mathrm{~m}$ long, $6.4 \mathrm{~m}$ wide, $4 \mathrm{~m}$ high and $80 \mathrm{~Hz}$ cut-off frequency). The floor is made of concrete and a $175 \mathrm{~mm}$ space separates it from the bottom of the cylinder. These acoustic conditions are supposed to weakly influence the shell vibrations as the bulk modulus of air is low (i.e. weak coupling between the fluid and the structure). The laser vibrometer is placed $2.68 \mathrm{~m}$ far from the cylindrical shell's surface as seen in Fig. 5. The vibrometer scans the cylinder from down to up along a single generating line. The experiment consists in keeping the vibrometer at a fixed position while rotating the set cylindrical shell-shaker.

The aim is to measure the radial vibrations on the outer surface of the cylindrical shell for the two configurations, that means with and without the non-axisymmetric internal frame. The measurements are made up to $16 \mathrm{kHz}$. The excitation is a chirp (swept-frequency sine), and the output is the transfer function with regard to the excitation force, calculated on 20 averages.

\subsection{Scanning grid definition}

The grid definition in the physical space determines the definition of the wavenumber domain (see the properties of discrete Fourier transform [21]). In order to examine the vibrations results in the wavenumber domain, the scanning grid is defined as a function of the flexural wavenumber $k_{f}$ of an equivalent plate 


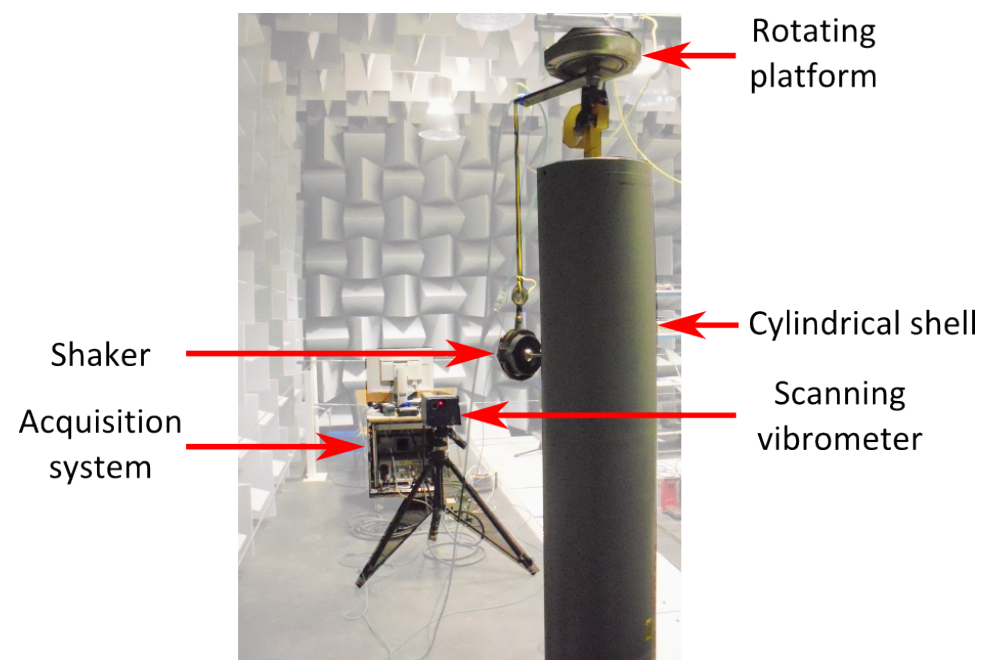

Figure 5: Picture of the experimental setup

(in steel, of thickness $h=1.5 \mathrm{~mm}$ ) at the highest frequency [18]:

$$
k_{f}=\left(\frac{\omega}{h} \sqrt{\frac{12\left(1-\nu^{2}\right) \rho_{s}}{E}}\right)^{1 / 2}
$$

To avoid aliasing effect, the maximum axial wavenumber is $k_{x}^{\max }=k_{f} \simeq$ $207 \mathrm{~m}^{-1}$ and the maximum circumferential number is $n_{\max }=k_{f} R_{0} \simeq 20$. For a cylindrical shell, as the system is stiffer than a plate because of the curvature, the natural wavenumbers of the propagative waves are lower than $k_{f}$. The criteria are thus stricter than they could be. The maximum distance $d_{\max }$ between two consecutive measurement points is then:

$$
d_{\max }=\frac{2 \pi}{2 k_{x}^{\max }}
$$

The numerical application yields $d_{\max }=15 \mathrm{~mm}$. A scanning line with $N_{x}=$ 101 points along the cylinder is thus defined. The maximum angle $\theta_{\max }$ between two consecutive measurements is given similarly by:

$$
\theta_{\max }=\frac{2 \pi}{2 n_{\max }}
$$

The present case gives $\theta_{\max }=9^{\circ}$ and results in $N_{\theta}=41$ lines to scan. The system being symmetric, it is sufficient to measure between 0 and $180^{\circ}$. The experiment consists then in 21 linear scans, where the system is successively rotated by $9^{\circ}$.

Similarly, the resolution in the wavenumber domain $\delta k_{x}$ is linked to the length of the cylindrical shell:

$$
\delta k_{x}=\frac{2 \pi}{L} \simeq 4.2
$$


The axial wavenumber resolution $\delta k_{x}$ can be enhanced using zero padding, but results show that the resolution is fine enough to observe the variations with the axial wavenumber.

\section{Operational dispersion curves for the axisymmetric system}

The 2D Fourier transform defined in Eq. (1) is applied to evaluate experimentally the velocity of the shell in the wavenumber domain. In practice, as the velocities have been measured on a regularly spaced grid, the integrals of Eq. 1) are approximated
the exponential functions:

$$
\tilde{\tilde{W}}\left(k_{x}, n\right) \simeq \sum_{i=1}^{N_{x}} \sum_{j=1}^{N_{\theta}} \dot{W}\left(x_{i}, \theta_{j}\right) e^{-\mathrm{j}\left(k_{x} x_{i}+n \theta_{j}\right)} d_{\max } \theta_{\max }
$$

where $\left(x_{i}, \theta_{j}\right)$ is the coordinate of a point on the scanning grid.

Operational dispersion curves are constructed by plotting the amplitude of the spectral radial velocity on the surface of the axisymmetric cylindrical shell as a function of the frequency and the axial wavenumber, for a given circumferential order. The plots in Fig. 6 show the dispersion curves for the circumferential orders $n=0, n=1, n=4$ and $n=6$ for the axisymmetric case. Theoretical models are presented in the left column and compared to the experimental results in the right column. The theoretical model uses the Fluegge equations for an infinite cylindrical shell with stiffeners included in the dynamic model using smeared theory [22, 23. An average stiffeners spacing of $20 \mathrm{~mm}$ is considered.

On the theoretical curves, the solid white line represents the flexural wavenumber for an equivalent plate projected on the circumferential order $n$ :

$$
k_{x f}(n)=\sqrt{k_{f}^{2}-\left(\frac{n}{R_{0}}\right)^{2}}
$$

where $k_{f}$ is the flexural wavenumber for a plate defined in Eq. (9). The dashed white line represents the acoustical wavenumber projected on the circumferential order $n$ :

$$
k_{x 0}(n)=\sqrt{k_{0}^{2}-\left(\frac{n}{R_{0}}\right)^{2}}
$$

The area under this dashed curve represents the supersonic domain, where the structural waves are strongly coupled to the acoustic domain and radiate efficiently. For the circumferential orders $n=0$ and $n=1$, the dashed-dotted 240 line respresents the longitudinal wavenumbers projected on the circumferential order $n$ given by:

$$
k_{x l}(n)=\sqrt{k_{l}^{2}-\left(\frac{n}{R_{0}}\right)^{2}}
$$




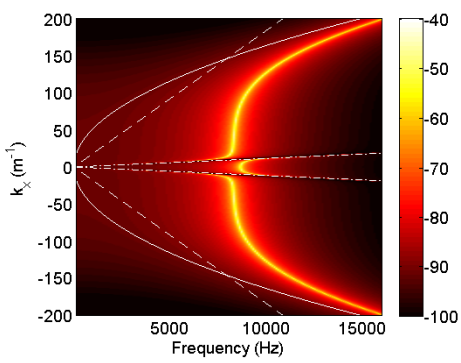

(a)

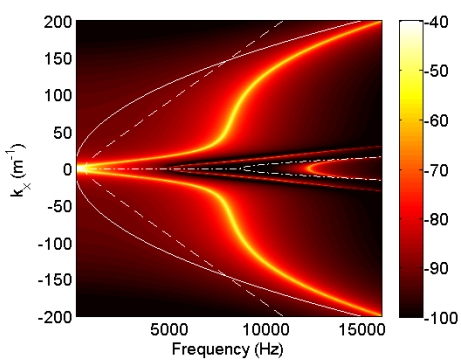

(c)

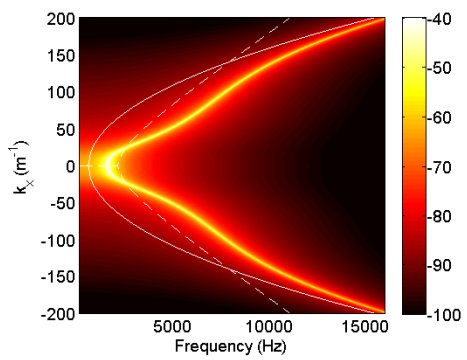

(e)

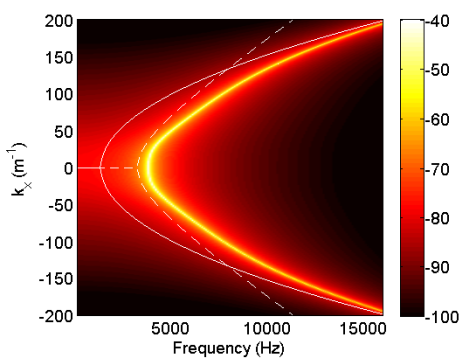

(g)

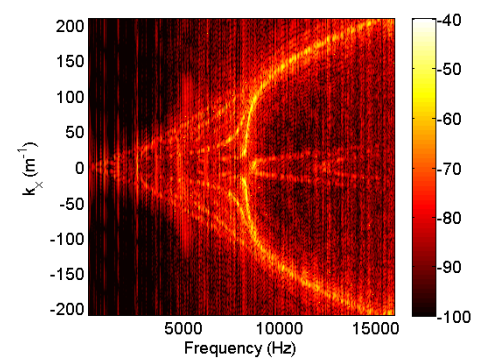

(b)

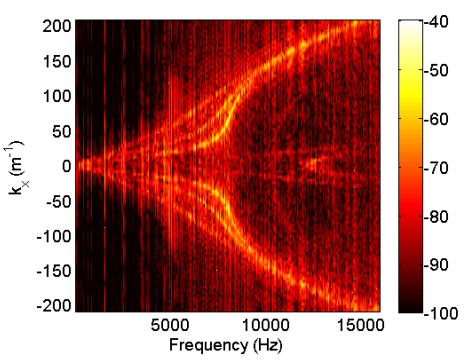

(d)

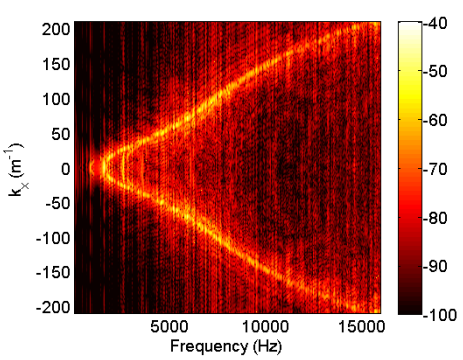

(f)

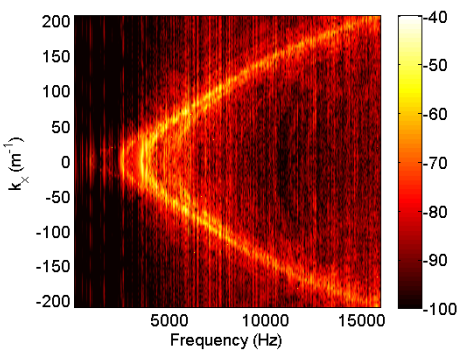

(h)

Figure 6: Operational dispersion curves plotted from the spectral radial velocities (dB ref $1 \mathrm{~m} . \mathrm{s}^{-1}$ ) in the axisymmetric case. (a) Theoretical, $n=0$. (b) Experimental, $n=0$. (c) Theoretical, $n=1$. (d) Experimental, $n=1$. (e) Theoretical, $n=4$. (f) Experimental, $n=4$. (g) Theoretical, $n=6$. (h) Experimental, $n=6$. 
where $k_{l}=\omega \sqrt{\rho_{s}\left(1-\nu^{2}\right) / E}$ is the longitudinal wavenumber. For the circumferential orders $n=4$ and $n=6, k_{x l}$ is not real and is not represented on the plots.

For the theoretical model in Fig. $6 \mathrm{a}$, the radial breathing mode $n=0$ is plotted. As expected, one can see that the model for a plate is not adapted at low frequencies (the solid white line does not fit the curve), meaning that the curvature of the cylindrical shell has an important effect on the vibroacoustic behavior. The trend for the plate model fits better at higher frequencies (above $25012 \mathrm{kHz}$ ), where the curvature can be neglected [24]. The breathing mode appears around $8.5 \mathrm{kHz}$, at the ring frequency [see Eq. (7)]. The coincidence frequency for the equivalent plate is given by the intersection of the flexural wavenumber curve with the acoustical wavenumber [see Eq. (8)] and reads around $7.9 \mathrm{kHz}$. This value is however not relevant for the present case because of the curvature. Indeed, as the operational dispersion curve from the cylindrical shell model lies always under the dashed curve, it shows that the breathing mode always radiates efficiently. In Fig. 6a and 6e, the curves seen close to the axis $k_{x}=0$ are propagating with high velocities. They are well approached by the dasheddotted lines and can be identified as longitudinal waves.

For the circumferential orders $n=1$ and $n=4$ in Fig. 6e and 6e respectively, the effect of curvature can also be seen. The dispersion curve have a S-shape instead of the square-root shape for the flexural waves in a plate. The coincidence frequency for the cylindrical shell model can then be read around $2.7 \mathrm{kHz}$, instead of $8 \mathrm{kHz}$ for the plate. Cut-on frequencies can also be seen on the operational dispersion curves. For instance, it can be seen that the waves cannot propagate for the circumferential order $n=4$ below $1500 \mathrm{~Hz}$. It can be checked that the cut-on frequency increases with the circumferential order. For the circumferential order $n=6$ in Fig. $6 \mathrm{~g}$, the cut-on frequency is around $3700 \mathrm{~Hz}$. For the circumferential order $n=6$, the effect of curvature is less 270 important and the dispersion curve is closer to the plate model. However, it can be seen that the flexural waves are faster than the plate model shown by the white solid line, due to added stiffness because of the curvature and the stiffeners. Consequently, the whole dispersion curve is in the supersonic domain and this circumferential order always radiates efficiently.

Comparing the experimental and theoretical operational dispersion curves in Fig. 6 shows a good agreement between the curves. On the experimental plots, spots of high amplitudes can be seen instead of continuous lines. These spots correspond to axial resonances due to the fact that the experimental cylindrical shell is of finite length. However, the comparison also shows that it is difficult to isolate the contribution from only one circumferential order in the experimental case. Additional dispersion curves can indeed be seen, even if their levels are lower than the main contribution. Three explanations are considered:

- As the cylindrical shell has sections with three different stiffeners spacings (see Fig. 1k), it could explain why several dispersion curves can be seen for one circumferential order. However, numerical tests show that the influence of the stiffeners spacing is weak on the dispersion curves and can 
only be seen at high frequencies.

- Uncertainties in the position of the points on the scanning grid can occur. These uncertainties can break the regularity of the measured velocity field and couple the circumferential orders. However, these uncertainties have been numerically tested on an infinite shell model and a equally distributed law on the points of the scanning grid (not shown here for the sake of conciseness). It can be seen that their influence is weak on the coupling of the circumferential orders.

- Although the particular experimental care, the system and the excitation are not perfectly axisymmetric. Indeed, it is really hard in practice to be sure that the shaker only excites the cylindrical shell in a perfectly radial direction. Besides there may be imperfections on the cylindrical shell geometry because of the machining $(0.1 \mathrm{~mm}$ tolerance on the shell thickness for instance) and the damages during transportation. It is known that non-axisymmetries tend to couple circumferential orders [13].

This suggests that the additional dispersion curves seen in the right column of Fig. 6 are a coupling of the circumferential orders due to imperfections in the axisymmetry of the system.

\section{Influence of the non-axisymmetric internal frame}

\subsection{Mean quadratic velocity}

The mean quadratic velocity defined in Eq. (6) is written in a discrete form as

$$
<\dot{W}>^{2}=\frac{1}{S} \sum_{i=1}^{N_{x}} \sum_{j=1}^{N_{\theta}}\left|\dot{W}\left(x_{i}, \theta_{j}\right)\right|^{2} d_{\max } R_{0} \theta_{\max }
$$

The mean quadratic velocity is plotted as a function of the frequency in Fig. 7 a

the axisymmetric case. Only the frequency range between 0 and $2500 \mathrm{~Hz}$ is shown in this plot in order to clearly see the resonance peaks. By looking at the velocity repartition on the surface of the cylindrical shell for the resonance peaks, one can notice that the circumferential modes appear by groups. This phenomenon is linked to the fact that there is a cut-on frequency below which the circumferential orders cannot appear, as explained in [25] and on the dispersion curves in section 4

Before placing the non-axisymmetric internal frame in the cylinder, it was hanged with flexible strings and an accelerometer was placed at an arbitrary spot in order to measure the frequency response to an impact hammer. The 320 transfer function is plotted in Fig. $7 \mathrm{~b}$. This plot allows estimating qualitatively the number of resonances of the isolated non-axisymmetric internal frame, which appears high in comparison with the number of resonances of the axisymmetric cylindrical shell in the frequency range considered ( $c f$. Fig. 7 a).

The comparison of the mean quadratic velocities for the two configurations 325 (with and without the non-axisymmetric internal frame) is plotted as a function 


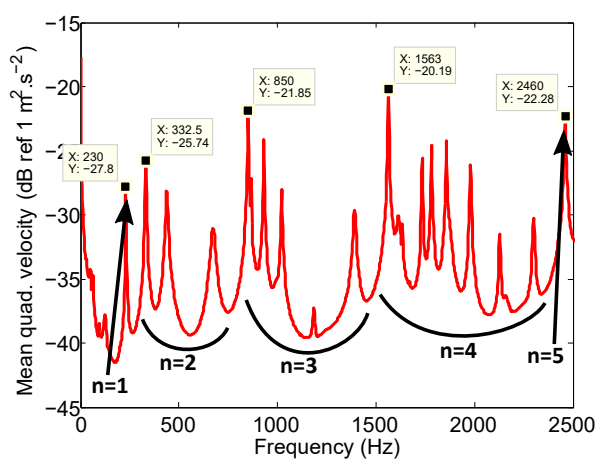

(a)

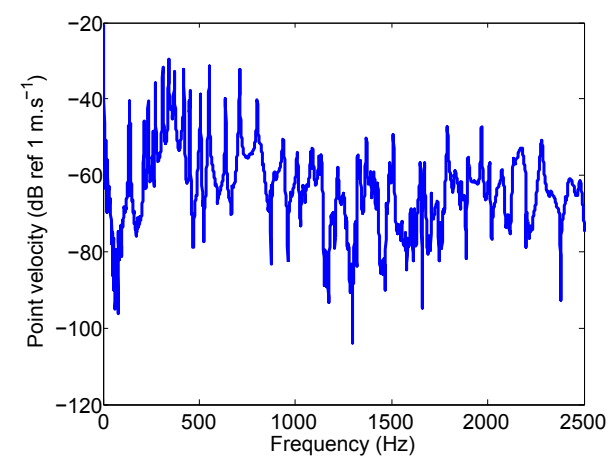

(b)

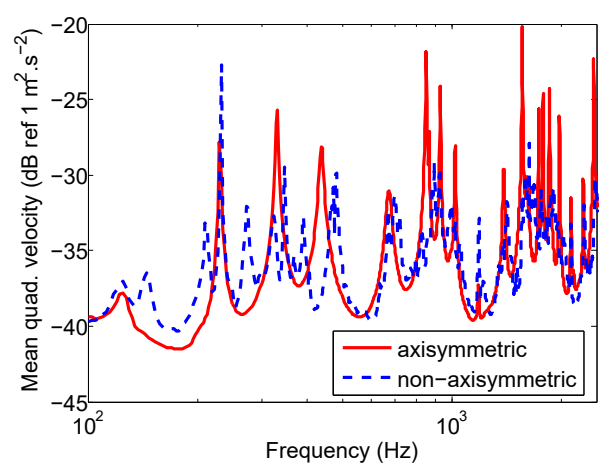

(c)

Figure 7: (a) Mean quadratic velocity as a function of the frequency for the axisymmetric stiffened cylindrical shell. (b) Transfer function between an impact point on the top of the floor and an accelerometer placed on the side. (c) Comparison of the mean quadratic velocities for the two configurations.

of the frequency in Fig. 77. The first observation is that the vibration level is lower in the non-axisymmetric case, in particular above $500 \mathrm{~Hz}$. The cylindrical shell and the internal structure have the same thickness and are made from the same material. We can expect that the mechanical impedances of the two structures are similar and then adapted to exchange vibratory energy from one structure to another. Besides, the modal density of the internal structure is higher than the one of the cylindrical shell. The combination of these two elements leads to an apparent damping effect on the shell because of energy being trapped in the internal frame. Moreover, new resonance peaks can be observed, in particular at low frequency between 200 and $500 \mathrm{~Hz}$. The peaks frequencies (at 213, 234 and $273 \mathrm{~Hz}$ for instance) are directly linked to the resonance of the non-axisymmetric internal frame seen in Fig. 7 b. 


\subsection{Radiated power and radiation efficiency}

The stationary phase theorem given in Eq. (3) is used to calculate the pressure radiated in the far-field using the vibrations measurements on the cylindrical shell in the wavenumber domain. In practice, the sum is calculated for $n$ between $-n_{\max }$ and $n_{\max }$. The far-field radiated power is deduced by discretization of Eq. (4):

$$
P_{a}=\frac{1}{\rho_{0} c_{0}} \sum_{i_{\xi}=1}^{N_{\xi}} \sum_{j_{\theta}=1}^{N_{\theta}} p^{2}\left(\mathcal{R}, \xi_{i}, \theta_{j}\right) \mathcal{R}^{2} \sin \theta_{j} \delta \xi \theta_{\text {max }}
$$

where $\left(\xi_{i}\right)_{i=1,2, \ldots, N_{\xi}}$ is the vector of azimuthal angles and $\delta \xi$ the step between two consecutive angles. For this example $\xi_{i}$ is varying between $-187^{\circ}$ and $+187^{\circ}$ with a step of $\delta \xi=3^{\circ}$

The radiated power calculated from the spectral displacements and the stationary phase theorem is plotted as a function of the frequency for the axisymmetric and non-axisymmetric case in Fig. $8 \mathrm{p}$. The influence of the nonaxisymmetric internal frame is mainly seen in the low frequencies (below $1 \mathrm{kHz}$ ). The radiation efficiency [see Eq. (5]] is plotted in Fig. 8b. The coincidence frequency for the equivalent plate $(h=1.5 \mathrm{~mm})$ is plotted with a vertical dotted line. It is clear that the non-axisymmetric internal frame tends to increase the radiation efficiency below the coincidence frequency. Differences up to $10 \mathrm{~dB}$

355 can be seen at some frequencies on this configuration. Above the coincidence frequency, it can be said that the structure radiates efficiently with or without the non-axisymmetry.

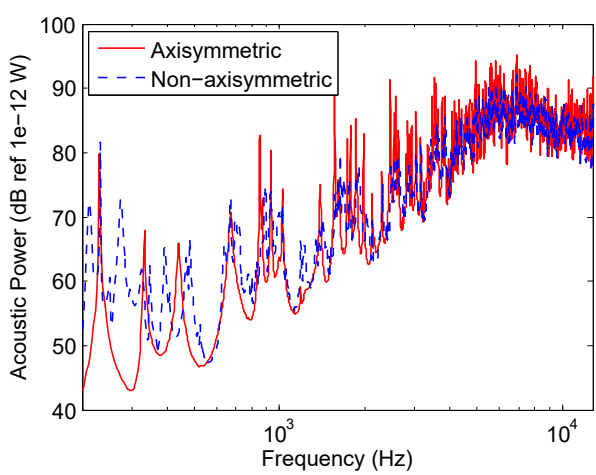

(a)

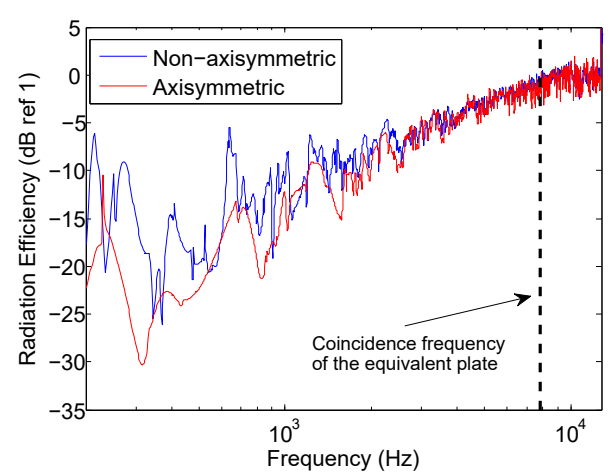

(b)

Figure 8: Estimation as a function of the frequency for the two configurations using the stationary phase theorem of (a) the radiated power ( $\mathrm{dB}$ ref $1 \mathrm{e}-12 \mathrm{~W})$ and $(\mathrm{b})$ the radiation efficiency (dB ref 1$)$.

To explain the increase in the radiation efficiency, a focus is done on the operational dispersion curves in the frequency range up to $3000 \mathrm{~Hz}$, where the radiation efficiency increases in the non-axisymmetric case. The experimental 
operational dispersion curves for this frequency range are plotted for the axisymmetric and non-axisymmetric case in Fig. 9 for the circumferential orders $n=0$ and $n=4$. It can be seen that in the non-axisymmetric case, the contribution of the circumferential order $n=0$ is higher than in the axisymmetric case, while the amplitude of the waves of circumferential order $n=4$ is lower. It means that there is a coupling between the circumferential orders because of the internal frame, and that energy is transmitted from higher circumferential orders to lower ones. Besides, it has been shown in Fig. 6at the circumferential order $n=0$ is efficiently radiating, even in the low frequency range. This explains why the cylindrical shell radiates more efficiently when it is coupled to a non-axisymmmetric internal frame. It confirms the observations from numerical simulations 12. In the next section, a closer look is taken at the velocity field to understand the influence of the non-axisymmetric internal frame on the repartition of the vibratory energy.

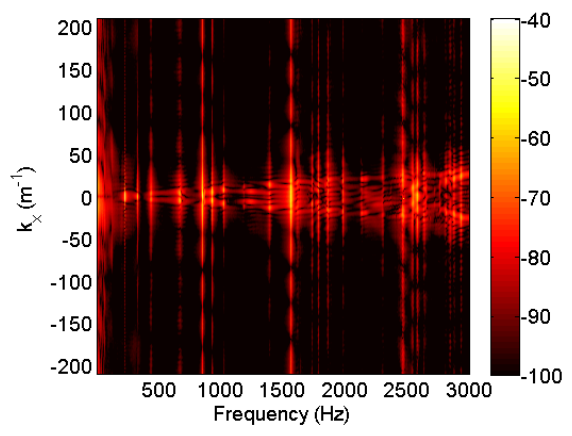

(a)

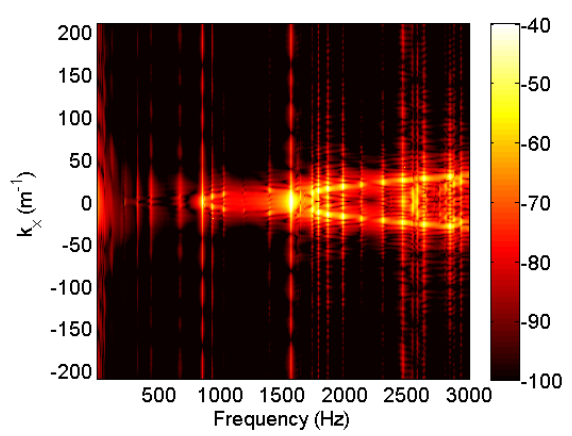

(c)

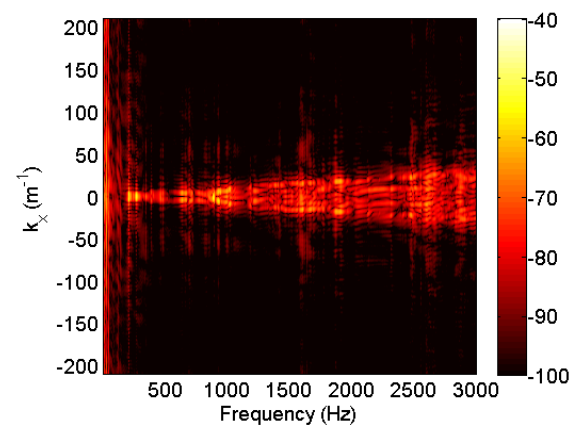

(b)

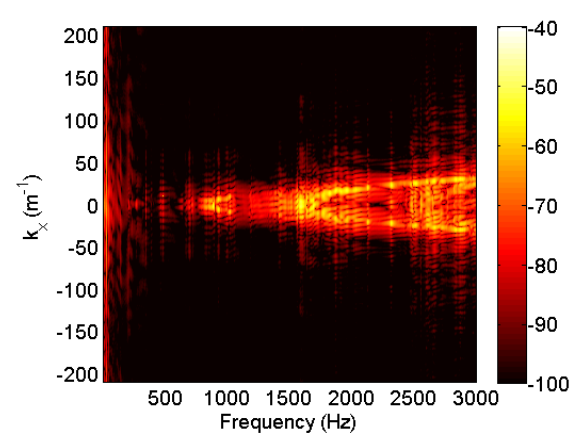

(d)

Figure 9: Operational dispersion curves plotted from the spectral radial velocities (dB ref 1 $\mathrm{m} . \mathrm{s}^{-1}$ ) in the low frequency range. (a) Axisymmetric case, $n=0$. (b) Non-axisymmetric case, $n=0$. (c) Axisymmetric case, $n=4$. (d) Non-axisymmetric case, $n=4$ 


\section{Maps of the radial vibratory field}

The velocity maps on the outer surface of the cylindrical shell are plotted in Fig. 10 for the axisymmetric and the non-axisymmetric configurations, at $f=1980 \mathrm{~Hz}$ and $f=3580 \mathrm{~Hz}$. These frequencies are chosen because they show an increase in the radiation efficiency in the non-axisymmetric case $(2.8 \mathrm{~dB}$ at $1980 \mathrm{~Hz}$ and $1.9 \mathrm{~dB}$ at $3580 \mathrm{~Hz}$ ) while having a drop in the radiated power (from $82.8 \mathrm{~dB}$ to $66.5 \mathrm{~dB}$ at $1980 \mathrm{~Hz}$ and from $82.5 \mathrm{~dB}$ to $80.4 \mathrm{~dB}$ at $3580 \mathrm{~Hz}$ ). Besides, energy transfer through the internal structure can be observed. Looking at the frequency response in Fig. $7 \mathrm{a}$, it can be said that the axisymmetric cylindrical shell has a resonance at $1980 \mathrm{~Hz}$ and has no resonance at $3580 \mathrm{~Hz}$ (out of the range in Fig. 7 a, but not on a resonance peak). The plots in Fig. 10 are functions of the tangential coordinate $\theta$ in abscissa and the axial coordinate $x$ in ordinate. The excitation is placed in $\theta=0$ and $x=1195 \mathrm{~mm}$. A spot of low velocities can be seen around the excitation, because the vibrometer cannot scan the surface behind the shaker. At $f=1980 \mathrm{~Hz}$, the mode $(m, n)=(8,4)$ is observed for the axisymmetric case, where $m$ is the longitudinal modal index and $n$ the circumferential order. Adding the non-axisymmetric internal structure has two main influences on the cartography at this frequency: the amplitude decreases and the nodes of vibrations are modified.

In Fig. 10, it can be seen that the point force mainly excites the area close to the excitation point. One of the possible explanations is that the cylindrical shell has sections with different stiffeners spacings. This means that there is a mechanical impedance discontinuity between two consecutive sections, which explains the localization effect seen at $f=3580 \mathrm{~Hz}$. In the non-axisymmetric case (Fig. 10 d), the energy can be transmitted through the internal structure 400 to the other end of the cylinder, where the stifeners spacing is the same than in the section which is excited (see Fig. 1 1 ).

To see the role played by the low circumferential orders, the radial velocities are plotted in the wavenumber space for the two configurations and the two frequencies in Fig. 11. In each plot, the amplitude has been normalized to the highest value in order to compare the repartition of the radial velocities in the wavenumber space. At $f=1980 \mathrm{~Hz}$, the amplitude maxima appear in the axisymmetric case at the wavenumbers corresponding to the modal wavenumbers. The circumferential order $n=4$ is playing a leading role in this case. In the non-axisymmetric case, more circumferential orders play a role, especially in the 410 low circumferential orders. The same observations can be made at $f=3580 \mathrm{~Hz}$ in Fig. 11k and 11d.

The radiation circle defined by Eq. (2) is plotted in white on the plots in Fig. 11. The radiation circles are elliptical due to the plotting aspect ratio. It can be noticed that the components inside the circle, which contribute to the far-field radiated pressure, have a higher relative level in the non-axisymmetric case. The radiation efficiency is thus expected to increase at these frequencies, as shown in section 5.2 . 


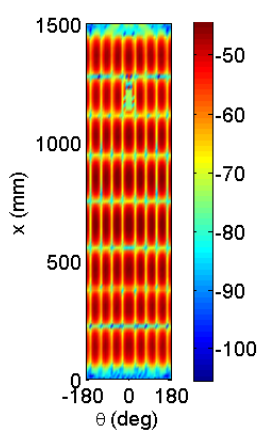

(a)

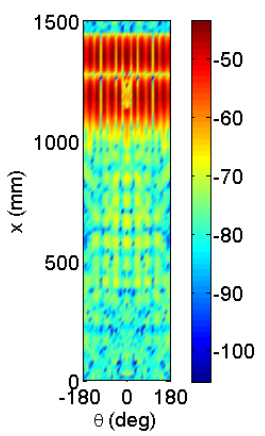

(c)

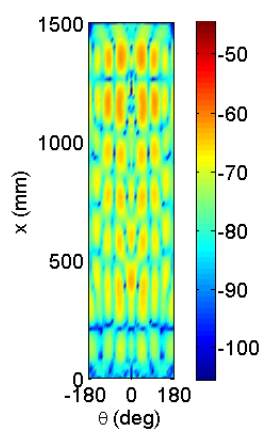

(b)

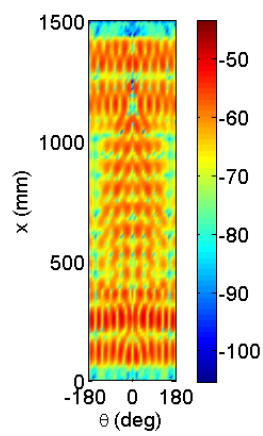

(d)

Figure 10: Map of radial velocities (dB ref $1 \mathrm{~m} \cdot \mathrm{s}^{-1}$ ) on the outer surface of the cylindrical shell. (a) Asymmetric case at $f=1980 \mathrm{~Hz}$. (b) Non-axisymmetric at $f=1980 \mathrm{~Hz}$. (c) Asymmetric case at $f=3580 \mathrm{~Hz}$. (d) Non-axisymmetric at $f=3580 \mathrm{~Hz}$.

\section{Conclusions}

Experiments have been conducted on a stiffened cylindrical shell to investigate the effect of a non-axisymmetric internal frame. A scanning laser vibrometer has been used to measure the radial velocities on a grid on the outer surface of the point-driven cylindrical shell, with and without a non-axisymmetric internal frame. A wavenumber analysis of the vibrations of the shell is used to discuss the vibroacoustic behavior of the shell. It shows that more circumferential orders tend to play a role when the cylindrical shell is coupled to the non-axisymmetric internal frame. More particularly energy is transmitted from high to low circumferential orders. As the low wavenumbers are in the supersonic domain, the structure tends to radiate more efficiently. This phenomenon explains the increase in radiation efficiency below the coincidence frequency seen 430 in the non-axisymmetric case.

Further work consists in conducting the same experimental process at higher frequencies. It will allow studying the influence of the non-axisymmetric internal 


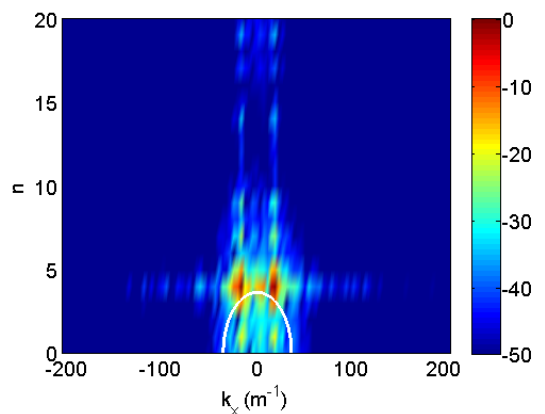

(a)

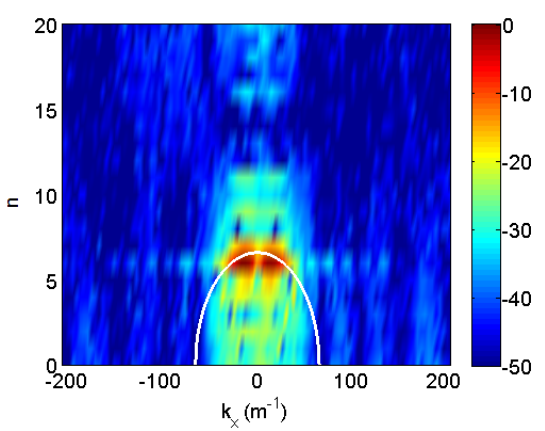

(c)

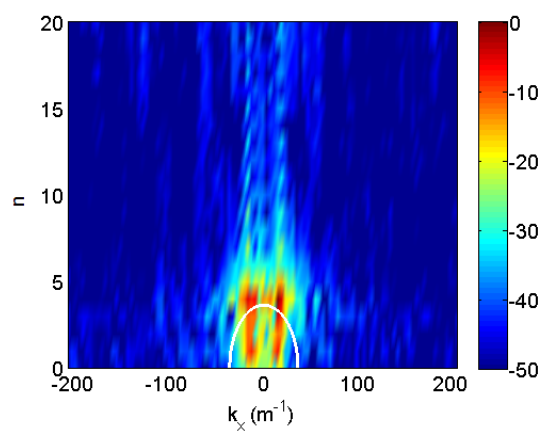

(b)

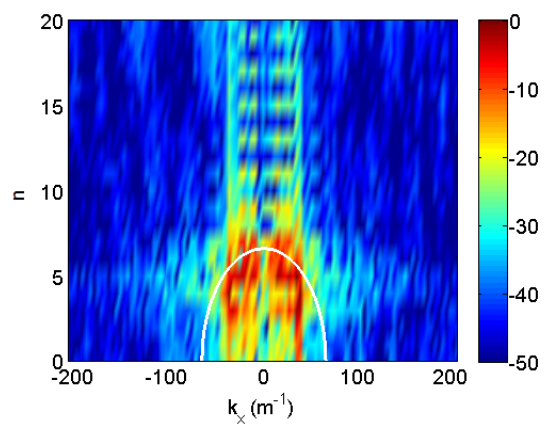

(d)

Figure 11: Map of radial velocities (dB ref $1 \mathrm{~m} \cdot \mathrm{s}^{-1}$ ) in the wavenumber domain $\left(k_{x}, n\right)$. (a) Asymmetric case at $f=1980 \mathrm{~Hz}$. (b) Non-axisymmetric at $f=1980 \mathrm{~Hz}$. (c) Asymmetric case at $f=3580 \mathrm{~Hz}$. (d) Non-axisymmetric at $f=3580 \mathrm{~Hz}$.

frame on the propagation of Bloch-Floquet waves due to periodic stiffeners. Besides, if the model is submerged in water, the coincidence frequency is about 20 times higher. In this case, it is likely that the effect of the internal frame is seen on a wider frequency range. Moreover, the strong coupling with the fluid can be investigated.

\section{Acknowledgment}

This work was funded by DCNS and performed within the framework of the ${ }_{440}$ LabEx CeLyA of Université de Lyon, operated by the French National Research Agency (ANR-10-LABX-0060/ANR-11-IDEX-0007). The authors are grateful to Patrick Blachier for his great contribution to the experimental setup. 


\section{References}

[1] R. N. Arnold, G. B. Warburton, Flexural vibrations of the walls of thin cylindrical shells having freely supported ends, Proceedings of the Royal Society of London. Series A. Mathematical and Physical Sciences 197 (1049) (1949) 238-256.

[2] G. Galletly, On the in-vacuo vibrations of simply supported, ring-stiffened cylindrical shells, Proceedings of the United States National Congress of Applied Mechanics 2 (1954) 225-231.

[3] W. H. Hoppmann II, Some characteristics of the flexural vibrations of orthogonally stiffened cylindrical shells, The Journal of the Acoustical Society of America 30 (1) (1958) 77-82.

[4] E. G. Williams, B. H. Houston, J. A. Bucaro, Broadband nearfield acoustical holography for vibrating cylinder, The Journal of the Acoustical Society of America 86 (2) (1989) 674-679.

[5] E. G. Williams, B. H. Houston, J. A. Bucaro, Experimental investigation of the wave propagation on a point-driven, submerged capped cylinder using k-space analysis, The Journal of the Acoustical Society of America 87 (2) (1990) 513-522.

[6] D. M. Photiadis, E. G. Williams, B. H. Houston, Wave-number space response of a near periodically ribbed shell, The Journal of the Acoustical Society of America 101 (2) (1997) 877-886.

[7] D. M. Photiadis, J. A. Bucaro, B. H. Houston, The effect of internal oscillators on the acoustic response of a submerged shell, The Journal of the Acoustical Society of America 101 (2) (1997) 895-899.

[8] D. M. Photiadis, B. H. Houston, E. G. Williams, J. A. Bucaro, Resonant response of complex shell structures, The Journal of the Acoustical Society of America 108 (3) (2000) 1027-1035.

[9] M. H. Marcus, B. H. Houston, The effect of internal point masses on the radiation of a ribbed cylindrical shell, The Journal of the Acoustical Society of America 112 (3) (2002) 961-965.

[10] H. Peters, R. Kinns, N. J. Kessissoglou, Effects of internal mass distribution and its isolation on the acoustic characteristics of a submerged hull, Journal of Sound and Vibration 333 (6) (2014) 1684-1697.

[11] M. M. Ettouney, R. P. Daddazio, N. N. Abboud, The interaction of a submerged axisymmetric shell and three-dimensional internal systems, International Journal for Numerical Methods in Engineering 37 (17) (1994) 2951-2970. 
[12] V. Meyer, L. Maxit, J.-L. Guyader, T. Leissing, Prediction of the vibroacoustic behavior of a submerged shell with non-axisymmetric internal substructures by a condensed transfer function method, Journal of Sound and Vibration 360 (2016) 260-276.

[13] E. Rebillard, B. Laulagnet, J.-L. Guyader, Influence of an embarked springmass system and defects on the acoustical radiation of a cylindrical shell, Applied Acoustics 36 (2) (1992) 87-106.

[14] C. Soize, Probabilistic structural modeling in linear dynamic analysis of complex mechanical systems, I-theoretical elements, La Recherche Aérospatiale (English edition) 5 (1986) Pages-23.

[15] A. D. Pierce, Resonant-frequency-distribution of internal mass inferred from mechanical impedance matrices, with application to fuzzy structure theory, Journal of vibration and acoustics 119 (3) (1997) 324-333.

[16] ISO, 3745, Acoustics - Determination of sound power levels of noise sources using sound pressure - Precision methods for anechoic and semi-anechoic rooms.

[17] ISO, 9614-2, Acoustics - Determination of sound power levels of noise sources using sound intensity - Part II: Measurement by scanning.

[18] M. C. Junger, D. Feit, Sound, structures, and their interaction, Vol. 240, MIT press Cambridge, MA, 1972.

[19] B. E. Sandman, Fluid-loading influence coefficients for a finite cylindrical shell, The Journal of the Acoustical Society of America 60 (6) (1976) 12561264 .

[20] J. E. Manning, G. Maidanik, Radiation properties of cylindrical shells, The Journal of the Acoustical Society of America 36 (9) (1964) 1691-1698.

[21] L. Maxit, Wavenumber space and physical space response of a periodically ribbed plate to a point drive: A discrete approach, Applied Acoustics 70 (2009) 563-578.

[22] M. Caresta, N. J. Kessissoglou, Acoustic signature of a submarine hull under harmonic excitation, Applied acoustics 71 (1) (2010) 17-31.

[23] A. Rosen, J. Singer, Vibrations of axially loaded stiffened cylindrical shells, Journal of Sound and Vibration 34 (3) (1974) 357-378.

[24] M. Heckl, Vibrations of point-driven cylindrical shells, The Journal of the Acoustical Society of America 34 (10) (1962) 1553-1557.

[25] J. Van de Loock, étude de la source de rayonnement acoustique des rsonances gnres dans une coque cylindrique partiellement immerge. application au domaine de l'olien offshore., Ph.D. thesis, Université du Havre (2015). 